\title{
Cognition and Emotion: The Cognitive Regulation of Emotions
}

\section{: A Review}

\author{
Mina Khatibi ${ }^{1}$, Dr. Farideh Yousefi ${ }^{2}$
}

Keywords: Cognition, Emotion

One of life's great challenges is successfully regulating emotions (Gross, 2002). The topic of emotion regulation has been of interest since Freud (1923) began to examine the relationship between the control of affective impulses and psychic health ( Krohne et al., 2002) . The emerging field of emotion regulation studies how individuals influence which emotions they have, when they have them, and how they experience and express them. Emotion regulation is defined and distinguished from coping, mood regulation, defense, and affect regulation (Gross, 1998). Many studies have been conducted in the field of cognition and emotion; e.g., emotion regulation: Past, present, future (Gross, 1999), the cognitive regulation of emotions: The role of success versus failure experience and coping dispositions (Krohne et al., 2002), the cognitive control of emotion (Ochsner and Gross, 2005), relationships between cognitive emotion regulation strategies and depressive symptoms: A comparative study of five specific samples, (Garnefski and Kraaij, 2002), incorporating emotion regulation into conceptualizations and treatments of anxiety and mood disorders (Campbell-Sills et al., 2007), healthy and unhealthy emotion regulation: Personality processes, individual differences, and life span development (John and Gross, 2004), emotion regulation in adulthood: Timing is everything (Gross, 2001 ),emotional states, attention, and working memory. Emotion regulation in depression: Relation to cognitive inhibition (Joormann and Gotlib, 2010), individual differences in two emotion regulation processes: Implications for affect, relationships, and well-being (Gross and John, 2003), mindfulness and emotion regulation: The development and initial validation of the Cognitive and Affective Mindfulness Scale-Revised (CAMS-R)(Laurenceau, 2007), regulation of distress and negative emotions: A developmental view (Kopp, 1989), basic emotions, relations among emotions, and emotion-cognition relations (Izard, 1992), emotional reactivity and cognitive regulation in anxious children, (Carthy, 2010), cognitive emotion regulation strategies and depressive symptoms: differences between males and females (Garnefski et al., 2004), cognitive emotion regulation in the prediction of depression, anxiety, stress, and anger (Martin and Dahlen, 2005), academic emotions in students' self-regulated learning and achievement: A

\footnotetext{
${ }^{1}$ PhD Student, Department of Educational Psychology, Shiraz University, Dubai, UAE ${ }^{2}$ Professor of Educational Psychology, Shiraz University, Shiraz, Iran

(C) 2015 I M Khatibi, F Yousefi; licensee IJIP. This is an Open Access Research distributed under the terms of the Creative Commons Attribution License (http://creativecommons.org/licenses/by/2.0), which permits unrestricted use, distribution, and reproduction in any Medium, provided the original work is properly cited.
} 


\section{Cognition and Emotion: The Cognitive Regulation of Emotions: A Review}

program of qualitative and quantitative research ( Pekrun et el., 2002), emotion regulation and mental health (Gross and Muñoz, 1995), specificity of cognitive emotion regulation strategies:

A transdiagnostic examination (Aldao and Nolen-Hoeksema, 2010), composure at any cost? The cognitive consequences of emotion suppression (Richards and Gross, 2013), now you feel It, now you don't, frontal brain electrical asymmetry and individual differences in emotion regulation (Jackson et al., 2003), community violence exposure and children's social adjustment in the school peer group: The mediating roles of emotion regulation and social cognition (Schwartz et al., 2000), emergent themes in the study of emotional development and emotion regulation (Campos et al., 1989), cognitive inhibition and emotion regulation in depression (Joormann, 2010). Contemporary conceptions of emotion emphasize emotions' positive role in adaptation (e.g., Lazarus, 1991; Tooby and Cosmides, 1990). Emotions are said to tailor cognitive style to situational demands (Clore, 1994), to facilitate decision-making (Oatley and Johnson-Laird, 1987), to prepare the individual for rapid motor responses (Frijda, 1986), and to promote learning (Cahill, 1994).

Historical Background: The study of emotion regulation has its origins in the psychoanalytic and stress and coping traditions. Research especially concerned with emotion regulation is a relatively recent innovation, but an interest in how emotions can and should be regulated is new (Averill, 1982). In the past century, this longstanding interest in emotion regulation has been played out in two principal arenas. Together, these have set the stage for contemporary research on emotion regulation.

REVIEW OF LITERATURE

Modern emotion theories emphasize the adaptive value of emotions. They often must be regulated. It is argued that distinctions need to be made between:

- Regulation of emotion and regulation by emotion;

- Emotion regulation in self and emotion regulation in others; and

- Conscious and nonconscious emotion regulation (Gross, 1999).

Krohne et al. (2002) concentrated on the analysis of two central cognitive mechanisms to regulate emotional states. Generating emotion-congruent or -incongruent cognitions and attention deployment (i.e., the regulation of emotions by shifting one's attentional focus.

The topic of emotion regulation has been of interest since Freud began to examine the relationship between the control of affective impulses and psychic health. Emotion regulation involves neurophysiological responses, the cognitive processes of attention, information processing, and encoding of internal cues, as well as behavioral mechanisms, such as response selection or regulating the demands of familiar settings (Gross, 1998; Thompson, 1994; Walden and Smith, 1997). 


\section{Cognition and Emotion: The Cognitive Regulation of Emotions: A Review}

Gross and Ochsner (2005) stated that the capacity to control emotion is important for human adaptation. Questions about the neural bases of emotion regulation have recently taken on new importance, as functional imaging studies in humans have permitted direct investigation of control strategies that draw upon higher cognitive processes difficult to study in nonhumans. Such studies have examined (1) controlling attention to, and (2) cognitively changing the meaning of, emotionally evocative stimuli.

Krohne et al. (2002) stated that attention deployment and generating specific types of cognitions are central cognitive mechanisms of emotion regulation. Two groups of hypotheses make contradicting predictions about the emotion-cognition relationship. The mood congruency hypothesis expects the emergence of mood-congruent cognitions (i.e., negative mood leads to negative and positive mood to positive cognitions). Similarly, a substantial body of research suggests that negative mood induces self-focus, whereas positive mood elicits an external focus of attention. In Krohne et al.'s study (2002), positive and negative emotional states were elicited by exposing the participants to the experience of success or failure in a demanding cognitive task. Cognitions that were present after emotion induction were assessed by means of a thoughtlisting procedure. For the total sample, results clearly confirmed the mood congruency hypothesis. Thought order was a critical factor only for changes in self-focus. Thought valence (positive, neutral, negative) as well as self-focus were substantially influenced by coping dispositions (Krohne et al., 2002).

According to Gross's process model of emotion regulation (Gross, 1998), strategies that act early in the emotion-generative process should have a different profile of consequences than strategies that act later on. His review focused on two commonly used strategies for downregulating emotion. The first, reappraisal, comes early in the emotion-generative process. The second, suppression, comes later in the emotion-generative process. Experimental and individual-difference studies find reappraisal is often more effective than suppression. Reappraisal decreases emotion experience and behavioral expression, and has no impact on memory. By contrast, suppression decreases behavioral expression, but fails to decrease emotion experience, and actually impairs memory. In his review, Gross concluded with a consideration of five important directions for future research on emotion regulation processes (Gross, 1998). According to Gross's process model of emotion regulation, emotion may be regulated at five points in the emotion generative process: (a) selection of the situation, (b) modification of the situation, (c) deployment of attention, (d) change of cognitions, and (e) modulation of responses. The field of emotion regulation promises new insights into age-old questions about how people manage their emotions (Gross, 1998).

Imaging studies have contributed to the development of a multilevel model of emotion regulation that describes the interactions between neural systems implicated in emotion generation and those implicated in emotional control. Ochsner et al. (2002) reviewed imaging studies of one type of cognitive emotion regulation: reappraisal. They showed that how imaging studies have contributed to the construction of this model, illustrated the interplay of 


\section{Cognition and Emotion: The Cognitive Regulation of Emotions: A Review}

psychological theory and neuroscience data in its development, and described how this model can be used as the basis for future basic and translational research (Ochsner et al., 2002).

Richards and Gross (2000) pointed out the affective consequences of emotion regulation. Little is known, however, about whether emotion regulation also has cognitive consequences. A process model of emotion suggests that expressive suppression should reduce memory for emotional events but that reappraisal should not. Three studies tested this hypothesis. Study 1 experimentally manipulated expressive suppression during film viewing, showing that suppression led to poorer memory for the details of the film. Study 2 manipulated expressive suppression and reappraisal during slide viewing. Only suppression led to poorer slide memory. Study 3 examined individual differences in typical expressive suppression and reappraisal and found that suppression was associated with poorer self-reported and objective memory but that reappraisal was not. Together, these studies suggest that the cognitive costs of keeping one's cool may vary according to how this is done (Richards and Gross, 2000).

Ochsner et al. (2002) stated that the ability to cognitively regulate emotional responses to aversive events is important for mental and physical health. Little is known, however, about neural bases of the cognitive control of emotion. Their study employed functional magnetic resonance imaging to examine the neural systems used to reappraise highly negative scenes in unemotional terms. Reappraisal of highly negative scenes reduced subjective experience of negative affect. Ochsner et al.'s findings support the hypothesis that prefrontal cortex is involved in constructing reappraisal strategies that can modulate activity in multiple emotion-processing systems (Ochsner et al., 2002).

Garnefski et al. (2001) described a new questionnaire, named the Cognitive Emotion Regulation Questionnaire, has been constructed, measuring nine cognitive coping strategies people tend to use after having experienced negative life events. A test-retest design was used to study the psychometric properties and relationships with measures of depression and anxiety among 547 high school youngsters. Cognitive coping strategies were found to play an important role in the relationship between the experience of negative life events and the reporting of symptoms of depression and anxiety. Garnefski et al.'s results suggested that cognitive coping strategies may be a valuable context of prevention and intervention (Garnefski et al., 2001).

Literature on the contributions of social cognitive and emotion processes to children's social competence is reviewed by Lemerise and Arsenio (2000) and interpreted in the context of an integrated model of emotion processes and cognition in social information processing.

Carstensen et al. (2003) pointed out that far more attention has been paid to emotion regulation in childhood than in adulthood and old age. However, a growing body of empirical research suggests that the emotion domain is largely spared from deleterious processes associated with aging and points instead to developmental gains in later life. Carstensen et al. attempted to explain the observed gains in terms of motivation. They argued that age is associated with increasing motivation to derive emotional meaning from life and decreasing 


\section{Cognition and Emotion: The Cognitive Regulation of Emotions: A Review}

motivation to expand one's horizons. These changes lead to age differences in social and environmental choices, coping, and cognitive processing of positive and negative information (Carstensen et al., 2003).

Berkowitz (1990) stated that noting a wide variety of unpleasant feelings, including sadness and depression, apparently can give rise to anger and aggression, he proposed a cognitive-neoassociationistic model to account for the effects of negative affect on the development of angry feelings and the display of emotional aggression.

Izard (1993) pointed out the significant role of emotions in evolution and adaptation suggesting that there must be more than 1 mechanism for generating them. The Izard's model described 4 types of emotion-activating systems, 3 of which involve noncognitive information processing. The emotion-activating systems operate under a number of constraints, including genetically influenced individual differences (Izard, 1993).

Research on expressive behavior has become well established in developmental, social, and personality psychology and in psychophysiology. Despite the success of the work on emotion expression and the upward trend of interest in emotions in general, the fundamental issue of the relation between emotion expression and emotion experience or feeling state remains controversia (Izard, 1990).

In losing control, Baumeister et al. (1994) provided a single reference source with comprehensive information on general patterns of self-regulation failure across contexts, research findings on specific self-control disorders, and commentary on the clinical and social aspects of self-regulation failure. Self-control is discussed in relation to what the self is, and the cognitive, motivational, and emotional factors that impinge on one's ability to control one's self (Baumeister et al., 1994).

Garnefski and Kraaij (2006) aimed to study relationships between cognitive emotion regulation strategies and depressive symptoms. Five specific samples, ranging from adolescents to elderly, were compared on their reported use of cognitive emotion regulation strategies (Rumination, Catastrophizing, Self-blame, Other-blame, Acceptance, Positive Reappraisal, Putting into Perspective, Positive Refocusing, Planning) and on the relationships between these strategies and symptoms of depression. Although remarkable differences were found in reported strategies, relationships between cognitive emotion regulation strategies and symptoms of depression were shown to be similar between the five groups (Garnefski and Kraaij, 2006).

Campbell-Sills and Barlow (2007) suggested that individual differences in emotion regulation may relate to vulnerability and resilience to anxiety and mood disorders. They also provided numerous examples of how many clinical features of anxiety and mood disorders may be construed as maladaptive attempts to regulate unwanted emotions. When using the term "emotion regulation," they referred to cognitive and behavioral processes that influence the occurrence, intensity, duration, and expression of emotion. These processes may support 


\section{Cognition and Emotion: The Cognitive Regulation of Emotions: A Review}

upregulation or downregulation of positive or negative emotions (Campbell-Sills and Barlow, 2007).

John and Gross (2004) focused on two commonly used emotion regulation strategies: reappraisal (changing the way one thinks about a potentially emotion-eliciting event) and suppression (changing the way one responds behaviorally to an emotion-eliciting event). First, they reviewed experimental findings showing that reappraisal has a healthier profile of shortterm affective, cognitive, and social consequences than suppression. Second, they reviewed individual-difference findings, which showed that using reappraisal to regulate emotions is associated with healthier patterns of affect, social functioning, and well-being than is using suppression. Third, they considered issues in the development of reappraisal and suppression and provided new evidence for a normative shift toward an increasingly healthy emotion regulation profile during adulthood (John and Gross, 2004).

Joormann and Gotlib (2010) aimed to identify cognitive processes that may be associated with the use of emotion-regulation strategies and to elucidate their relation to depression. Depression has been found to be associated with difficulties in cognitive control and, more specifically, with difficulties inhibiting the processing of negative material. They used a negative affective priming task to assess the relations among inhibition and individual differences in the habitual use of rumination, reappraisal, and expressive suppression in clinically depressed, formerly depressed, and never-depressed participants. They found that depressed participants exhibited the predicted lack of inhibition when processing negative material. Moreover, within the group of depressed participants, reduced inhibition of negative material was associated with greater rumination. Joormann and Gotlib concluded that these findings suggest that individual differences in the use of emotion-regulation strategies play an important role in depression, and that deficits in cognitive control are related to the use of maladaptive emotion-regulation strategies in this disorder (Joormann and Gotlib, 2010).

Laurenceau (2007) aimed to develop a brief self-report measure of mindfulness with items that cover the breadth of the construct and that are written in everyday language. The resulting 12-item measure demonstrated acceptable internal consistency and evidence of convergent and discriminant validity with concurrent measures of mindfulness, distress, wellbeing, emotion-regulation, and problem-solving approaches in three samples of university students (Laurenceau, 2007).

The means used by young children to control their own emotions has received scant attention in the developmental literature. However, this competency represents an important aspect of developmental growth. Kopp (1989) emphasized regulation of distress and negative emotions. It focused on (a) the principles that underlie regulation of distress and negative

emotions among infants and young children and (b) developmental trends that occur during the first few years of life (Kopp, 1989). 


\section{Cognition and Emotion: The Cognitive Regulation of Emotions: A Review}

Garnefski et al. (2004) focused on comparability of men and women in (a) the extent to which they use specific cognitive emotion regulation strategies in response to the experience of life stress and (b) the extent to which the use of these strategies is related to the reporting of depressive symptoms. Significant differences were found in the strategies Rumination, Catastrophizing and Positive refocusing: women reported to use these strategies more often than men. However, no differences were found in the extent to which specific cognitive strategies were related to the reporting of depressive symptomatology. In both groups, higher extents of reporting self-blame, rumination and/or catastrophizing as strategies were strongly related to higher depression scores, whereas higher extents of using positive reappraisal were related to lower depression scores (Garnefski et al., 2004).

Cognitive coping processes have long been implicated in the experience and expression of emotion. Recently, a new instrument, the cognitive emotion regulation questionnaire (Garnefski et al., 2001), was developed to measure nine different cognitive coping strategies people often use when faced with a negative event: self-blame, other blame, rumination, catastrophizing, acceptance, putting into perspective, positive refocus, refocus on planning, and positive reappraisal.

Martin and Dahlen (2005) explored the relationships between the CERQ and depression, anxiety, stress, and anger. Results supported the convergent and discriminant validity of the CERQ and demonstrated that, independent of respondent gender, self-blame, rumination, catastrophizing, and positive reappraisal were among the most valuable predictors of negative emotions (Martin and Dahlen, 2005).

Pekrun et al. (2002) pointed out that in 5 qualitative studies, it was found that students experience a rich diversity of emotions in academic settings. Anxiety was reported most often, but overall, positive emotions were described no less frequently than negative emotions. Based on the studies in their article, taxonomies of different academic emotions and a self-report instrument measuring students' enjoyment, hope, pride, relief, anger, anxiety, shame, hopelessness, and boredom (Academic Emotions Questionnaire [AEQ]) were developed. Results showed that academic emotions are significantly related to students' motivation, learning strategies, cognitive resources, self-regulation, and academic achievement, as well as to personality and classroom antecedents. The findings indicated that affective research in educational psychology should acknowledge emotional diversity in academic settings by addressing the full range of emotions experienced by students at school and university (Pekrun, et al., 2002).

Gross (1999) considered that the study of emotion regulation has its origins in the psychoanalytic and stress and coping traditions. Recently, increased interest in emotion regulation has led to crucial boundary ambiguities that now threaten progress in this domain. It is argued that distinctions need to be made between (1) regulation of emotion and regulation by emotion; (2) emotion regulation in self and emotion regulation in others; and (3) conscious and nonconscious emotion regulation. Using a process model of emotion generation, his review 


\section{Cognition and Emotion: The Cognitive Regulation of Emotions: A Review}

considered promising research on basic processes and individual differences in emotion regulation and poses five theoretical challenges (Gross, 1999).

Gross and Muñoz (1995) in their article argued that emotion regulation is an essential (and traditionally underemphasized) feature of mental health. To develop this idea, they first defined the terms emotion, emotion regulation, and mental health. They then charted the development of emotion regulation and described its role in various facets of normal functioning. Then they considered what happens when emotion becomes dysregulated in a major depressive episode. They concluded by speculating about the role of emotion regulation in the broader context of public mental health (Gross and Muñoz, 1995).

Aldao and Nolen-Hoeksema (2010) stated that despite growing interest in the role of regulatory processes in clinical disorders, it is not clear whether certain cognitive emotion regulation strategies play a more central role in psychopathology than others. Similarly, little is known about whether these strategies have effects transdiagnostically. They examined the relationship between four cognitive emotion regulation strategies (rumination, thought suppression, reappraisal, and problem-solving) and symptoms of three psychopathologies (depression, anxiety, and eating disorders) in an undergraduate sample. Maladaptive strategies (rumination, suppression), compared to adaptive strategies (reappraisal, problem-solving), were more strongly associated with psychopathology and loaded more highly on a latent factor of cognitive emotion regulation. In addition, this latent factor of cognitive emotion regulation was significantly associated with symptoms of all three disorders (Aldao and Nolen-Hoeksema, 2010).

\section{REFERENCES}

Aldao, A., Nolen-Hoeksema, S. (2010). Specificity of cognitive emotion regulation strategies: A transdiagnostic examination. Behaviour Research and Therapy, 48, 10, 974-983.

Averill, J.R. (1982). Anger and aggression: An essay on emotion. New York: Springer.

Baumeister, R. F., Heatherton, T. F., Tice, D. M. (1994). Losing control: How and why people fail at self-regulation. San Diego, CA, US: Academic Press. xi 307 pp.

Berkowitz, L. (1990). On the formation and regulation of anger and aggression: A cognitiveneoassociationistic analysis. American Psychologist, 45(4), 494-503.

Cahill, L., Prins, B., Weber, M., Mc Gaugh, J.L. (1994). b-Adrenergic activation and memory for emotional events. Nature, 371, 702-704.

Campbell-Sills, L., Barlow, D. H., Gross, J. J. (Ed) (2007). Incorporating Emotion Regulation into Conceptualizations and Treatments of Anxiety and Mood Disorders. Handbook of Emotion Regulation. New York, NY, US: Guilford Press, pp. 542-559.

Campos, J.J., Campos, R .G. Barrett, K .C. (1989). Emergent themes in the study of emotional development and emotion regulation. Developmental Psychology, 25, 394-402.

Carstensen, L.L., Fung, H.H., Charles, S.T. (2003). Socioemotional selectivity theory and the regulation of emotion in the second half of life. Motivation and Emotion, 27, 2, 103-123. 


\section{Cognition and Emotion: The Cognitive Regulation of Emotions: A Review}

Carthy, T., Horesh, N., Apter, A., Edge, M.D., Gross, J.J. (2010). Emotional reactivity and cognitive regulation in anxious children. Behav Res Ther, 48(5), 384-93.

Clore, G. L. (1994). Why emotions vary in intensity. In P. Ekman and R. J. Davidson Eds., The nature of emotion: Fundamental questions pp. 386-393. New York: Oxford University Press.

Frijda, N.H. (1986). The emotions. Cambridge, U K: Cambridge University Press.

Garnefski, N., Kraaij, V. (2006). Relationships between cognitive emotion regulation strategies and depressive symptoms: A comparative study of five specific samples. Personality and Individual Differences, 40, 8, 1659-1669.

Garnefski, N., Teerds, J., Kraaij, V., Legerstee, J., van den Kommer, T. (2004). Cognitive emotion regulation strategies and depressive symptoms: Differences between males and females. Personality and Individual Differences, 36, 267-276.

Garnefski, N., van den Kommer, T., Kraaij, V., Teerds, J., Legerstee, J., Onstein, E. (2002). The relationship between cognitive emotion regulation strategies and emotional problems. European Journal of Personality, 16, 403-420.

Gross, J. J. (2001). Emotion regulation in adulthood: Timing is everything. Current Directions in Psychological Science, 10, 214-219.

Gross, J.J. (1998). Antecedent- and response-focused emotion regulation: Divergent consequences for experience, expression, and physiology. J. Pers. Soc. Psychol. 74, 224 237.

Gross, J.J. (1998). The emerging field of emotion regulation: an integrative review. Rev. Gen. Psychol. 2, 271-299.

Gross, J.J. (1999). Emotion regulation: Past, present, future, 13 (5), 551-573.

Gross, J.J. (2002). Emotion regulation: Affective, cognitive, and social consequences. Psychophysiology 39, 281-291.

Gross, J.J. and John, O.P. (2003). Individual differences in two emotion regulation processes: Implications for affect, relationships, and wellbeing. J. Pers. Soc. Psychol. 85, 348-362.

Gross, J.J. and Munoz, R .F. (1995). Emotion regulation and mental health. Clinical Psychology: Science and Practice, 2, 151-164.

Izard, C. E. (1992). Basic emotions, relations among emotions, and emotion-cognition relations. Psychological Review, 99(3), 561-565.

Izard, C. E. (1993). Four systems for emotion activation: Cognitive and noncognitive processes. Psychological Review, 100(1), 68-90.

Jackson, D.C., Mueller, C.J., Dolski, I., et al. (2003). Now you feel it, now you don't: Frontal brain electrical asymmetry and individual differences in emotion regulation. Psychol. Sci. 14, 612-617.

John, O.P. and Gross, J.J. (2004). Healthy and unhealthy emotion regulation: Personality processes, individual differences, and life span development. Journal of Personality, 72, 6, 1301-1334.

Joormann, J. and Gotlib, I.H. (2010). Emotion regulation in depression: Relation to cognitive inhibition. Cognition and Emotion, 24, 2, 281-298. 


\section{Cognition and Emotion: The Cognitive Regulation of Emotions: A Review}

Kopp, C. B. (1989). Regulation of distress and negative emotions: A developmental view. Developmental Psychology, 25(3), 343-354.

Krohne, H.W., Pieper, M., Knoll, N., Breimer,N. (2002). The cognitive regulation of emotions: The role of success versus failure experience and coping dispositions. Cognition and Emotion, 16 (2), 217-243.

Laurenceau, J.P. (2007). Mindfulness and emotion regulation: The development and initial validation of the Cognitive and Affective Mindfulness Scale-Revised (CAMS-R). Journal of Psychopathology and Behavioral Assessment, 29, 3, 177-190.

Lazarus, R. S. (1991). Progress on a cognitive-motivational-relational theory of emotion. American Psychologist, 46, 819-834.

Lemerise, E.A. and Arsenio, W.F. (2000). An integrated model of emotion processes and cognition in social information processing. Developmental Psychology, Child Development, 71, 1, 107-118.

Martin, R.C., Dahlen, E.R. (2005). Cognitive emotion regulation in the prediction of depression, anxiety, stress, and anger. Personality and Individual Differences, 39, 12491260.

Oatley, K. and Johnson -Laird, P.N. (1987). Towards a cognitive theory of emotions. Cognition and Emotion, 1, 29-50.

Ochsner, K.N., Ray, R.D., Jeffrey C. Cooper, C.C., et al. (2002). Rethinking feelings: An FMRI study of the cognitive regulation of emotion. J. Cogn. Neurosci. 14, 1215-1229.

Pekrun, R., Goetz, T., Titz, W., Perry, R.P. (2002). Academic emotions in students' selfregulated learning and achievement: A program of qualitative and quantitative research. Educational Psychologist, 37, 2, 91-105.

Richards, J. M. and Gross, J. J. (2000). Emotion regulation and memory: The cognitive costs of keeping one’s cool. Journal of Personality and Social Psychology, 79, 410-424.

Schwartz, D., Proctor, L. J. (2000). Community violence exposure and children's social adjustment in the school peer group: The mediating roles of emotion regulation and social cognition. Journal of Consulting and Clinical Psychology, 68(4), 670-683.

Thompson, R .A. (1994). Emotion regulation: A theme in search of definition. In N.A. Fox (Ed.), the development of emotion regulation: Biological and behavioral considerations. Monographs of the Society for Research in Child Development, 59, 25-52.

Thompson, R.A. (1994). Emotion regulation: A theme in search of definition. Monographs of the Society for Research in Child Development, 59, 2-3, 25-52.

Tooby, J. and Cosmides, L. (1990). The past explains the present: Emotional adaptations and the structure of ancestral environments. Ethology and Sociobiology, 11, 375-424.

Walden, T.A. and Smith, M.C. (1997). Emotion regulation. Motivation and Emotion, 21, 7-25. 